\title{
Effectiveness of Emotion-Focused Therapy on Emotional Dysregulation, Hopelessness and Suicidal Ideation in Post-Traumatic Stress Disorder Veterans
}

\section{ART ICLE INF O}

\section{Article Type}

Original Research

\section{Authors}

Mikaeili N. ${ }^{1} P h D$,

Molavi P. ${ }^{2} P h D$

Einy $\mathrm{S}^{*} M S C$,

Tagavy R. ${ }^{3} M S c$

How to cite this article
Mikaeili N, Molavi P, Einy S,
Tagavy R. Effectiveness of moti-
on-Focused Therapy on Emotion-
al Dysregulation, opelessness and
Suicidal Ideation in Post-Traum-
atic Stress Disorder Veterans.
Iranian Journal of War \& Public
Health. 2017;9(3):111-117.

*Psychology Department, Educational Sciences \& Psychology Faculty, University of Mohaghegh Ardabili, Ardabil, Iran

${ }^{1}$ Psychology Department, Educational Sciences \& Psychology Faculty, University of Mohaghegh Ardabili, Ardabil, Iran

${ }^{2}$ Psychiatry Department, Medical Faculty, Ardabil University of Medical Sciences, Ardabil, Iran

${ }^{3}$ Psychology Department, Psychology Faculty, Ardabil Branch, Islamic Azad University, Ardabil, Iran

\section{Correspondence}

Address: Psychology and Educational Sciences Faculty, End of Daneshgah Street, University of Mohaghegh Ardabili, Ardabil, Iran

Phone: +98 (45) 33262678

Fax: +98 (45) 33511508

sanaz.einy@yahoo.com

\section{Article History}

Received: January 29, 2017

Accepted: March 11, 2017

ePublished: July 27, 2017

\section{A B S T R A C T}

Aims Post-Traumatic Stress Disorder (PTSD) is one of the prominent psychological consequences of war that is seen in veterans. Emotion-focused therapy is a short-term intervention that can target a range of disruptions to the disorder. The purpose of this study was to investigate the efficacy of emotion-focused therapy on emotional distress, suicidal ideation and disappointment of veterans with PTSD.

Materials \& Methods In this semi-experimental design with pretest-posttest design with control group in year 2016, 50 veterans with PTSD who were referred to Isfahan Ardabil psychiatric hospital were selected through convenient sampling and randomly assigned to two groups of experimental and control groups. The data were collected using a post-traumatic stress disorder checklist-military version, Desperate Beck Questionnaire, Beck Suicidal Thinking Scale, and Threat Settlement Scale. Then, the sessions focused on excitement were performed for 8 sessions of 1.5 hours for the experimental group. Data were analyzed by SPSS 23 software using multivariate covariance analysis test.

Findings After controlling the effects of pretest, there was a significant difference between the mean posttest scores of the two groups in each of the emotional maladaptive, suicidal ideation and disappointment variables $(\mathrm{p}<0.05)$.

Conclusion Emotion-focused therapy can reduce emotional distress, suicidal thoughts and frustration of veterans with PTSD.

Keywords Dysthymic Disorder; Emotional Dysregulation; Suicidal Ideation; Emotion-Focused Therapy

\section{I T A T I O N L I NKS}

[1] Diagnostic and statistical manual ... [2] A preliminary investigation of ... [3] Emotion regulation in psychotherapy ... [4] Emotional processing of fear ... [5] The relationship of metacognitive ... [6] Emotion regulation difficulties as ... [7] Relationships between posttraumatic ... [8] Aria Pooran S. The effectiveness of ... [9] Attachment styles and cognitive ... [10] Post-traumatic stress disorder and suicide ... [11] A meta-analysis of the association ... [12] Strain, depressed mood and suicidal ... [13] Intimate partner violence, psychological distress ... [14] Posttraumatic stress disorder and suicide risk .. [15] The effectiveness of cognitive- existential group ... [16] Hope theory: rainbows ... [17] Insight into severe mental illness ... [18] The relationship of hope and quality ... [19] Cognitive therapy techniques ... [20] Exposure inhibition therapy as a treatment for ... [21] IPT for women with trauma histories in ... [22] The relation of rigidity across relationship ... [23] The emotions [24] Differential effects of emotion-focused ... [25] Emotion-focused therapy: The trans- forming power ... [26] The contribution of emotion processes to narrative ... [27] Emotion focused therapy for ... [28] Systems of ... [29] Task analysis exemplified: The process of resolving ... [30] Working with the emotions in ... [31] The treatment of combat trauma in veterans using EFT (Emotional Freedom Techniques) ... [32] Psychological trauma symptom improvement ... [33] Effectiveness of emotion focused therapy on sexual ... [34] The effects of emotion-focused couple therapy ... [35] Effectiveness of emotion regulation training ... [36] The effectiveness of cognitive self-compassion ... [37] Sport psychology uro ... [37] Clinician-administered PTSD scale ... [38] Investigation of clinical diagnosis by psychological ... [39] Reliability and validity of post ... [40] The measurement of pessimism ... [41] Conformity and norm of the beck ... [42] The relationship between acceptation ... [43] Interview and psychological ... [44] Multidimensional assessment of emotion regulation ... [45] The relationship emotional regulation ... [46] Emotion-Focused The rapy ... [47] The effectiveness of cognitive therapy ... [48] Problem- solving skills in suicidal psychiatric ... [49] The effectiveness of emotion ... 


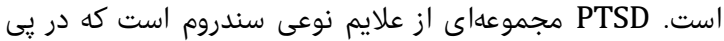

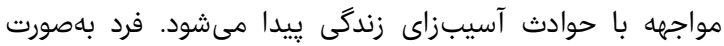

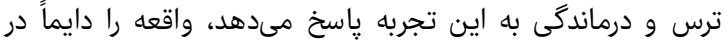

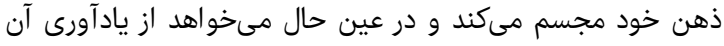

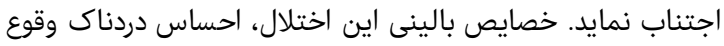

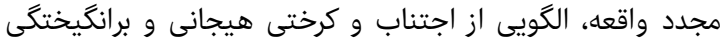

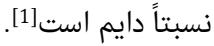

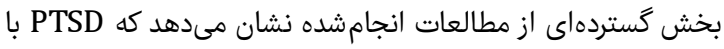

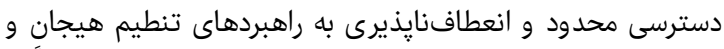

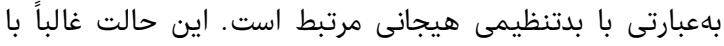

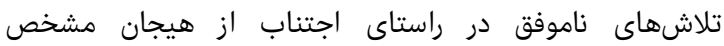

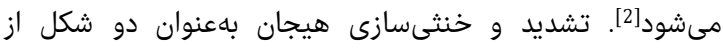

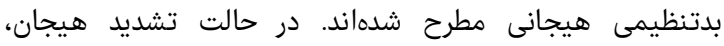

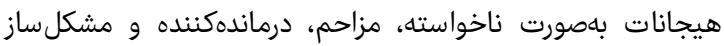

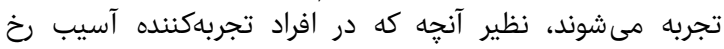

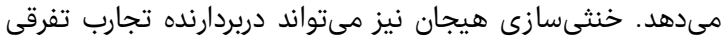

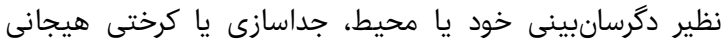

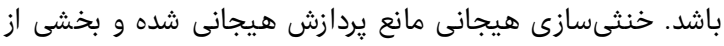

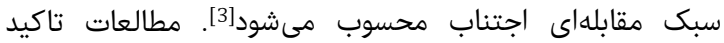

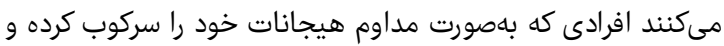

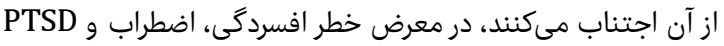

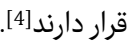

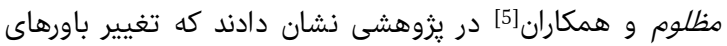

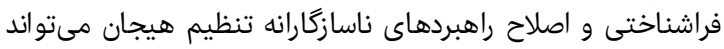

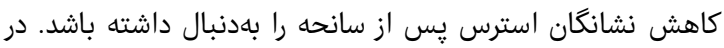

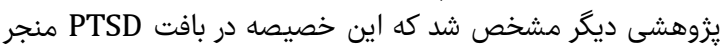

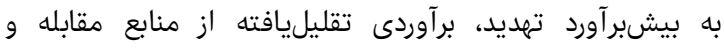

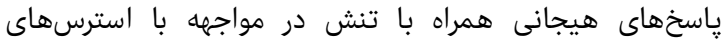

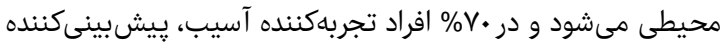

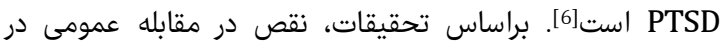

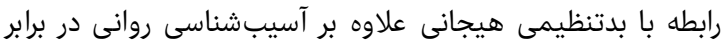

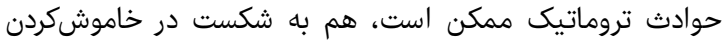

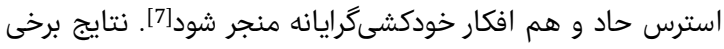

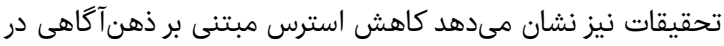

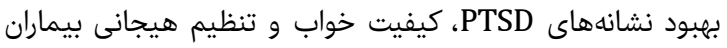

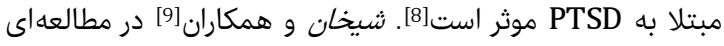

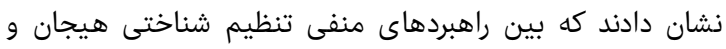

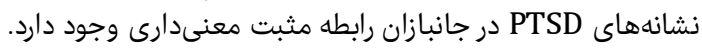

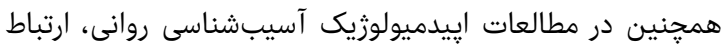

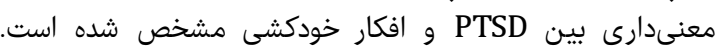

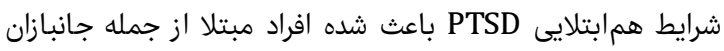

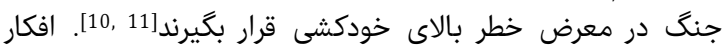

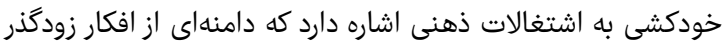

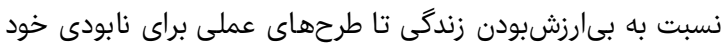

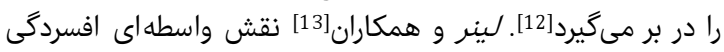

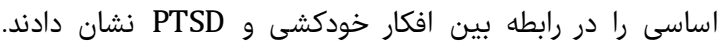

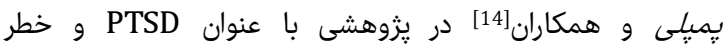

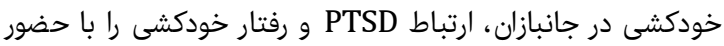

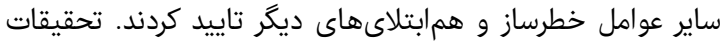

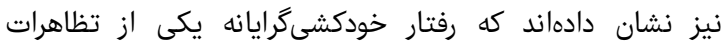

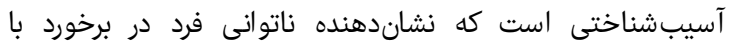
موقعيتهاى استرسزا همراه با اختلال در تنظيم هيجان نان است درد دران. دوره 9، شماره س، تابستان عوسا

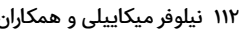

اثربخشى درمان متمركز بر هيجان بران بر بدتنظيمى إنى

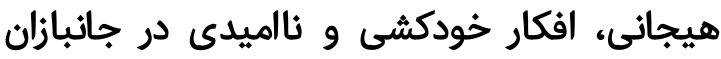

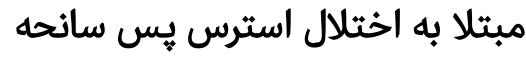

PhD نيلوفر ميكاييلى

كرّه روان شناسى، دانشكده علوم تربيتى و روان شناسى، دانشكاه محقق اردبيلى،

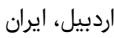

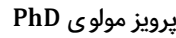

كروه اعصاب و روان، دانشكده يزشكى، دانشكاه علوم يزشكى اردبيل، اردبيل،

ايران

MSc " ساناز عينى

كَروه روانشناسى، دانشكده علوم تربيتى و روان شناسى، دانشكاه محقق اردبيلى، اردبيل، ايران

MSc رامين تقوى اريران

كرّه روانشناسى، دانشكده روانشناسى، واحد اردان اردبيل، دانشكاه آزاد اسلامى،

اردبيل، ايران

קكيده

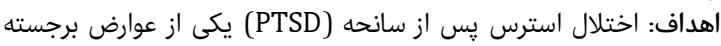

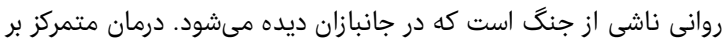

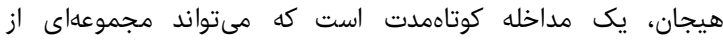

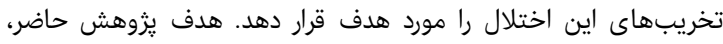

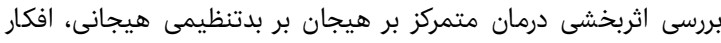

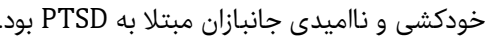

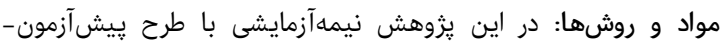

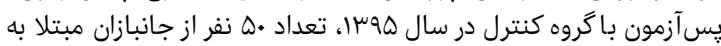

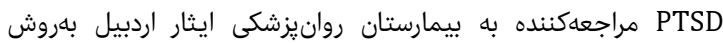

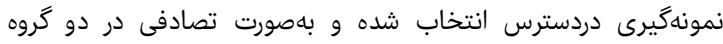

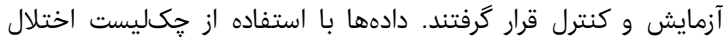

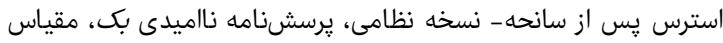

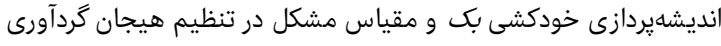

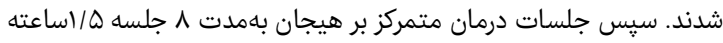

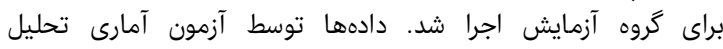

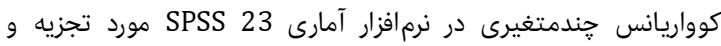

تحليل قرار گرفتند.

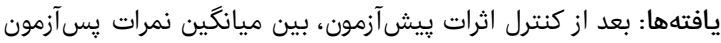

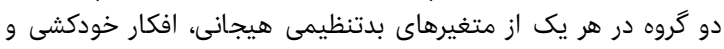

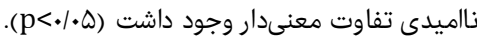

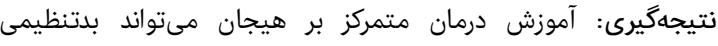

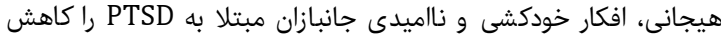

دهد.

كليدوازمها: درمان متمركز بر هيجان، بدتنظيمى هيجانى، افكار خودكشى، ناميدى،

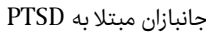

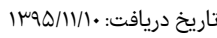

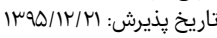

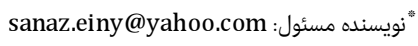

مقدمه

جنگ جنها از عوامل فشارآور روانى در زندكى نوين هستند. در طول جنگ عراق عليه ايران، اختلال استرس يس إن از از سانحه (PTSD)

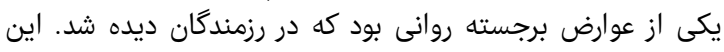

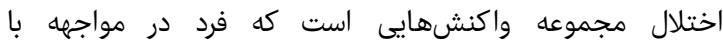

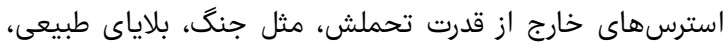

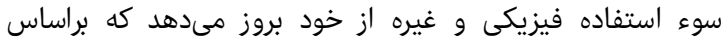

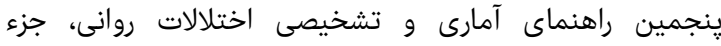
مجموعه تشخيصى اختلالات مرتبط با آسيب و وامنمي عامل استرسزا

فصل امه علمى - هُروهشى طب جانباز 


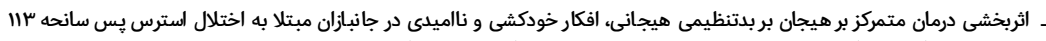

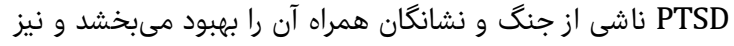

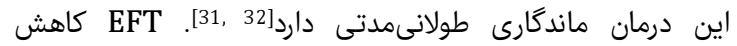

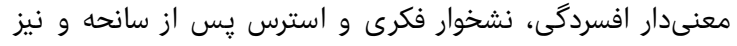

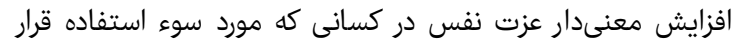

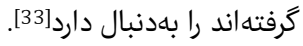

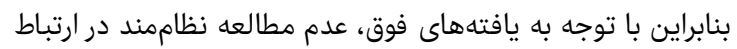

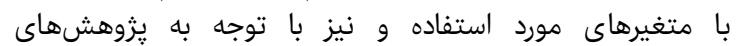

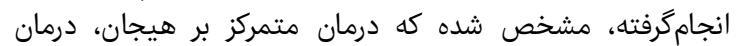

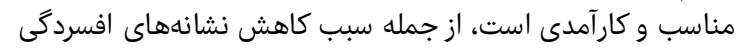

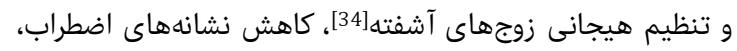

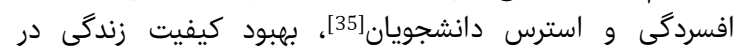

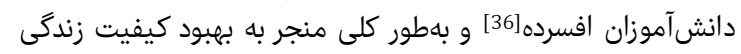

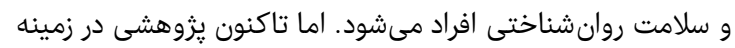

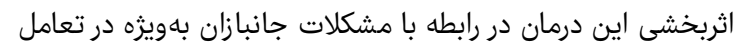
با هيجانات انجام نكُفته است درمان.

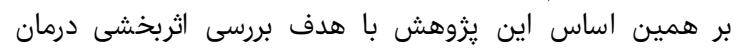

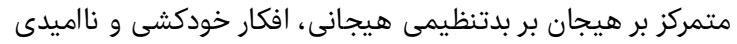

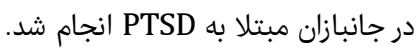

\section{مواد و روشها}

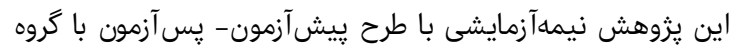

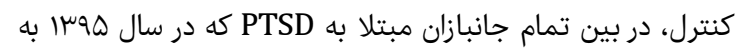

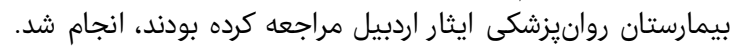

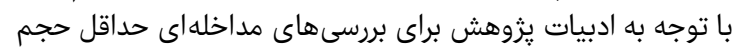

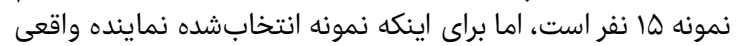

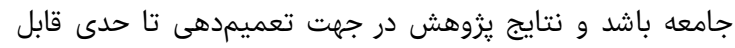

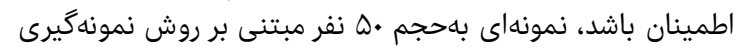

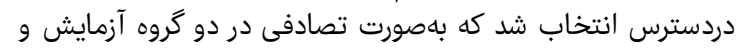
كنترل قرار كرفتند.

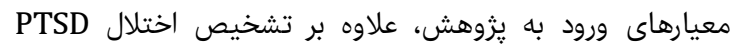

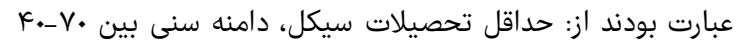

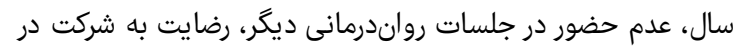

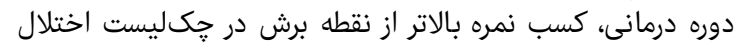

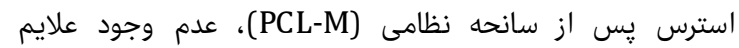

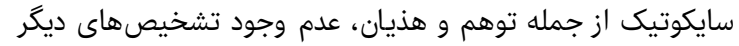

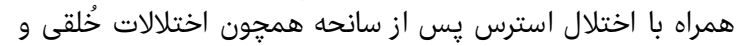

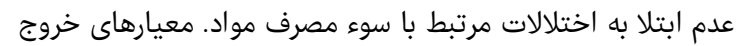

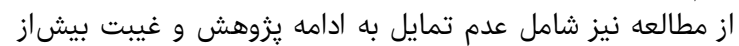

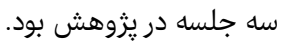

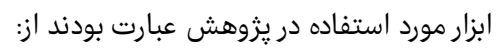

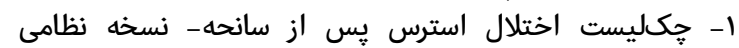
براس (PCL-M)

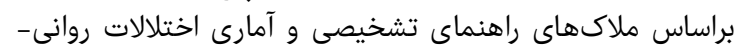

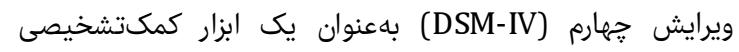

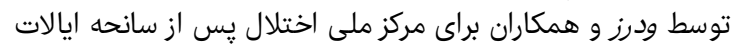

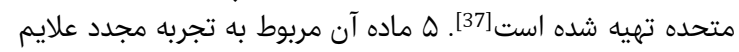

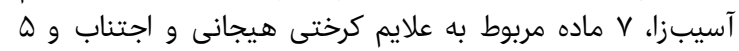

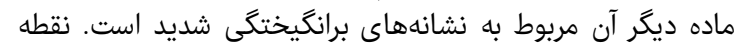

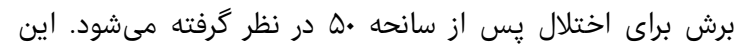

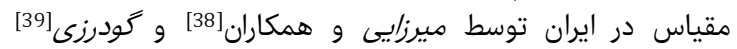

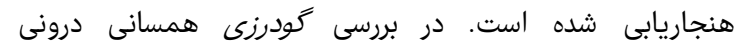

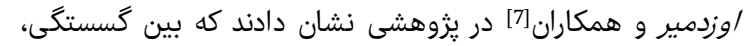

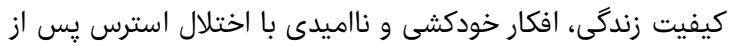
سانحه رابطه معنىدارى وجود دارئ دارد.

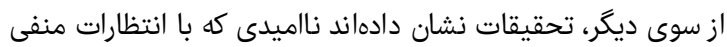
همراه است در PTSD و خودكشى نقش قابل ملاحظهاى داردان دان.

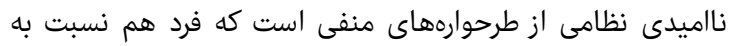

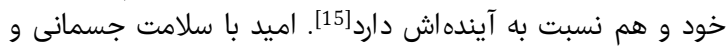

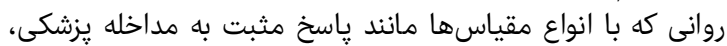

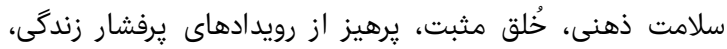

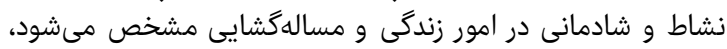

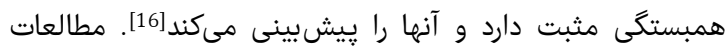

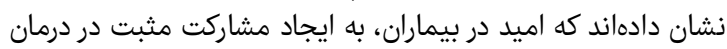

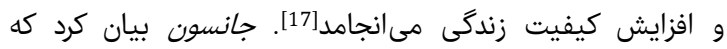

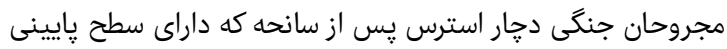

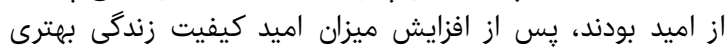

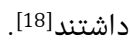

با توجه به اينكه هر سه سازه مورد نظر بهنوعى با هيجان مرتبط

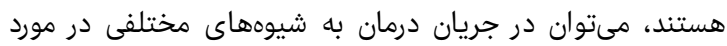

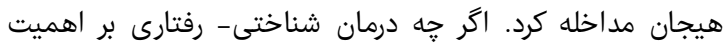

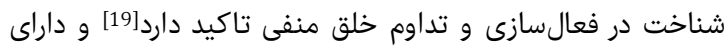

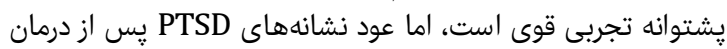

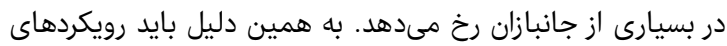

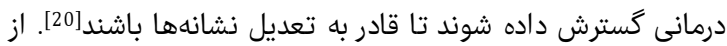

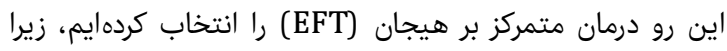

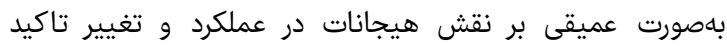

مىكند]21].

EFT

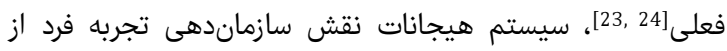

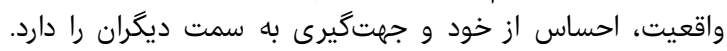
EFT

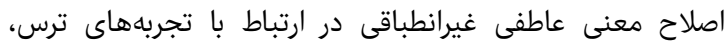

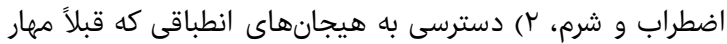

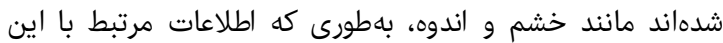

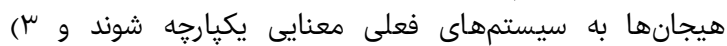

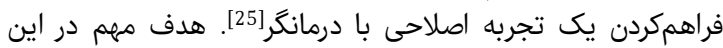

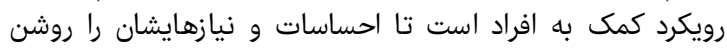

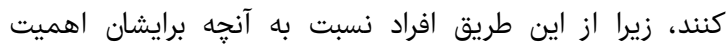

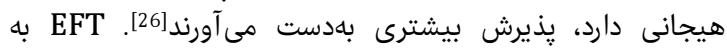

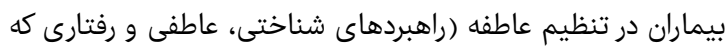

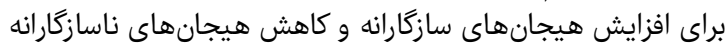

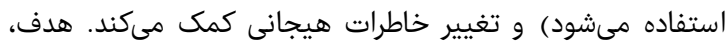

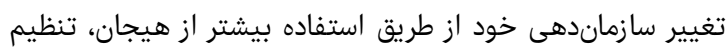

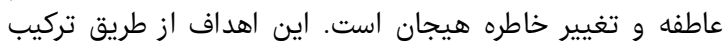

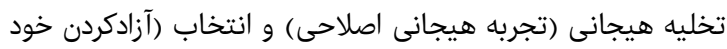

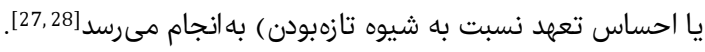

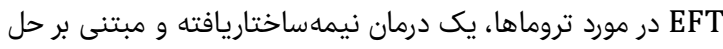

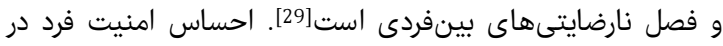

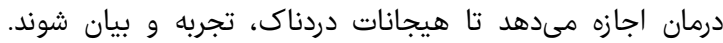

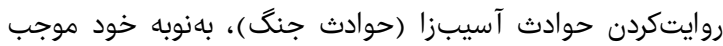

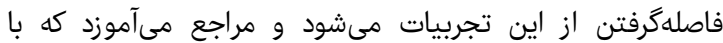

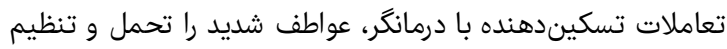

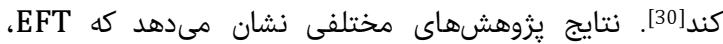

Volume 9, Issue 3, Summer 2017 
دوهفتهاى هـ/• كزارش كردند. پايايى نسخه فارسى اين مقياس

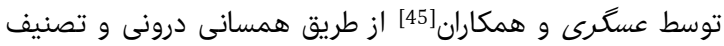

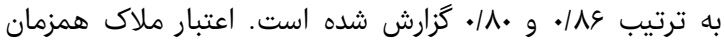

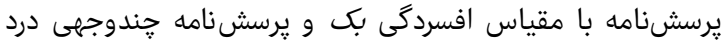
تاييد شده است] [45].

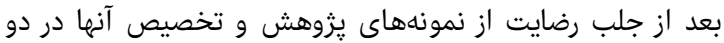

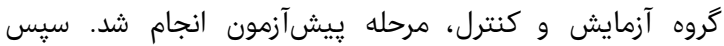

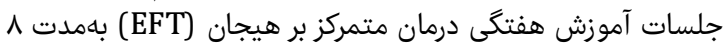

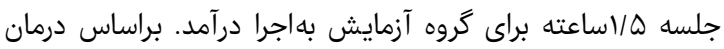

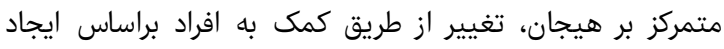

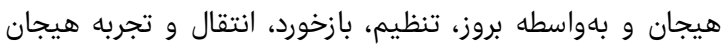

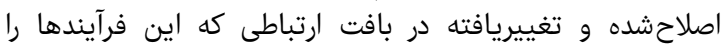

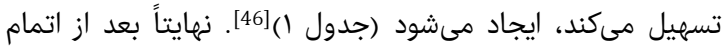

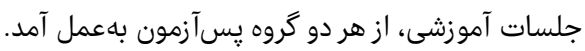

جدول ( ) محتواى جلسات درمان متمركز بر هيجان جلسه اول

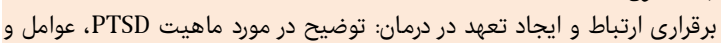

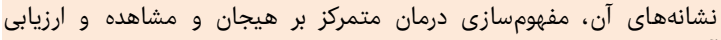

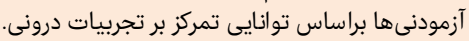

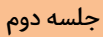

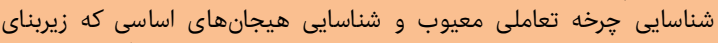

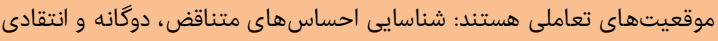

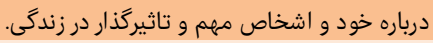

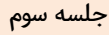

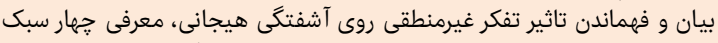

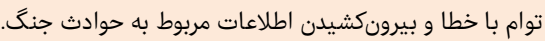

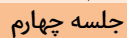

"ييوستكى و آكاهى، شناسايى فرآيندهاى شناختى عاطفى زيربنايى، شناسايى شرايط ايجاد مشكل.

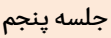

آرامسازى عضلانى، شناسايى طرحوارههاى هيجانى، تاكيد بر يذيرش تجائ تجربيات،

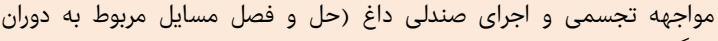
جنَ) (جن).

جلسه ششم

برجستهاسلى و شرح مجدد واقعه جنكَ، اشاره به طبيعىبودن آن، تحليل رويا (بهمنظور كاهش كابوس با با اصول متمركز بر هيجانات). جلسه هفتم

تقويت يردازش هيجانى، رديابى احساسات حلنشداته، بازسازى مجانى مجدد هيجان،

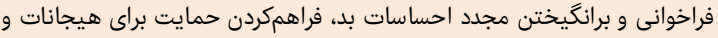

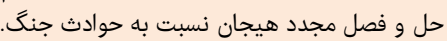
جلسه هشتم

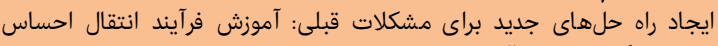

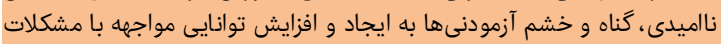

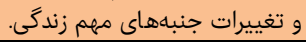

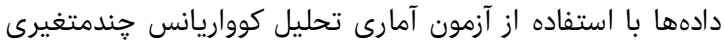

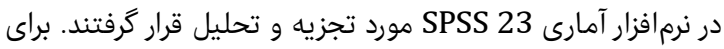

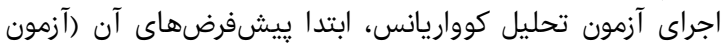

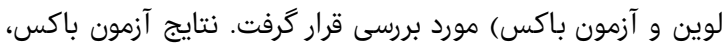

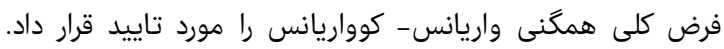

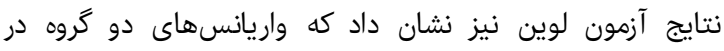

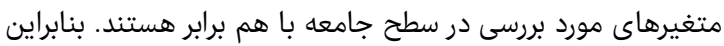

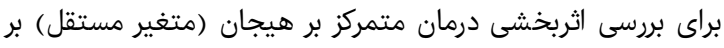

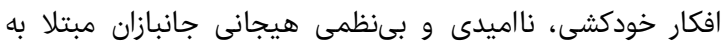
PTSD

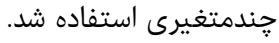

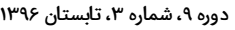

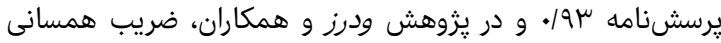

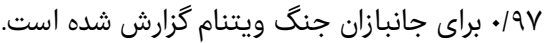

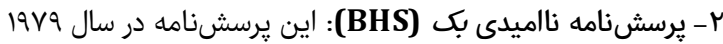

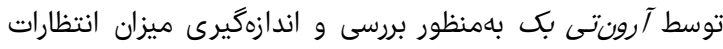

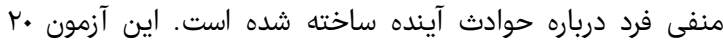

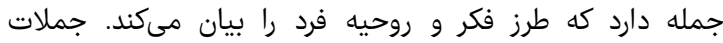

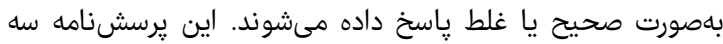

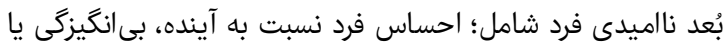

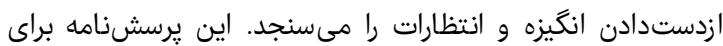

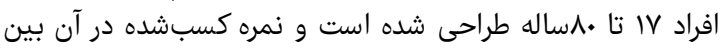

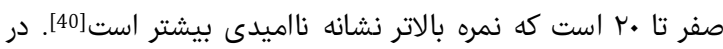

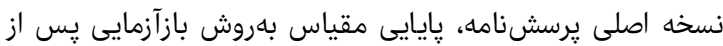

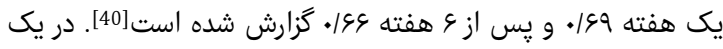

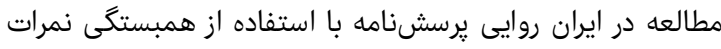

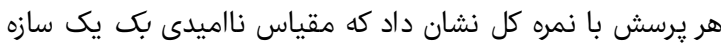

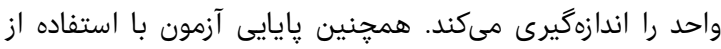

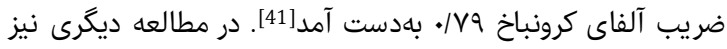

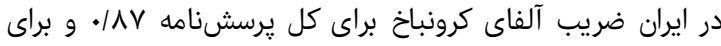

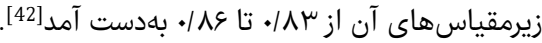

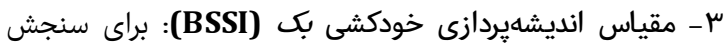

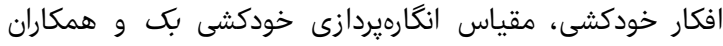

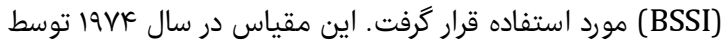

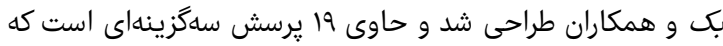

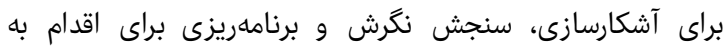

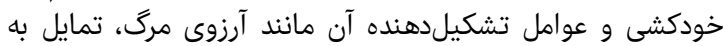

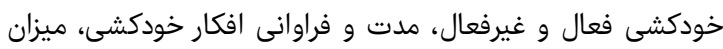

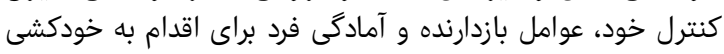

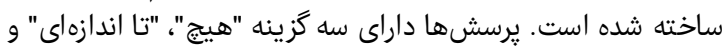

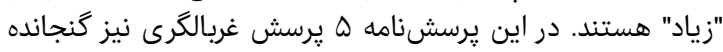

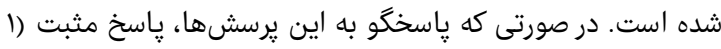

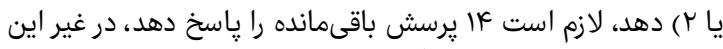

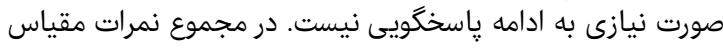

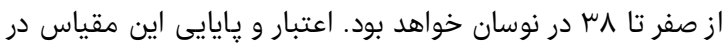

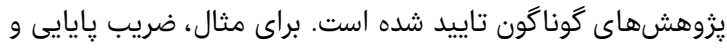

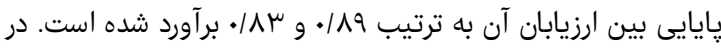

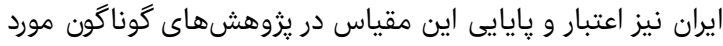

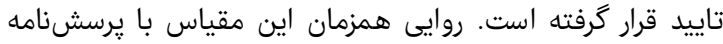

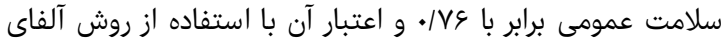

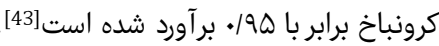

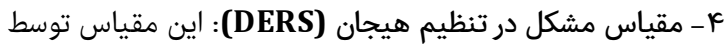

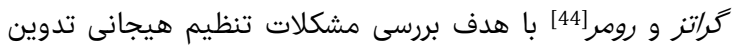

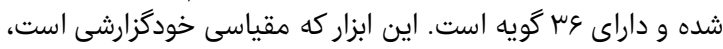

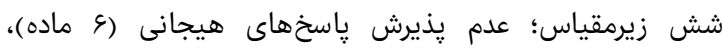

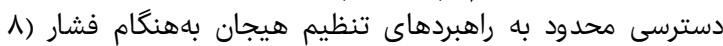

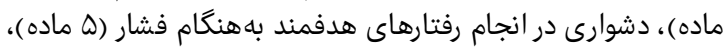

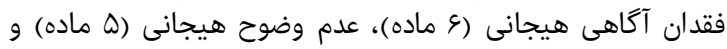

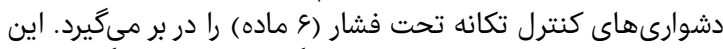

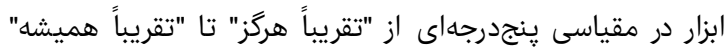

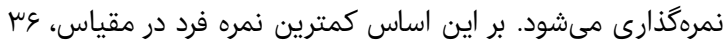

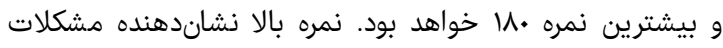

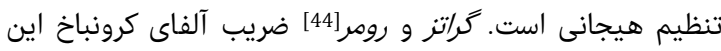

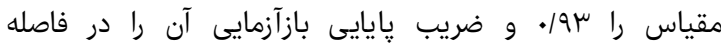




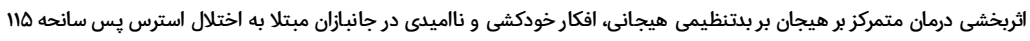

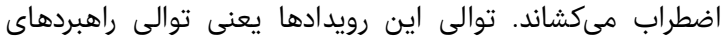

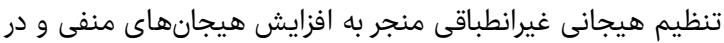

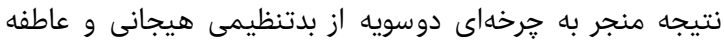

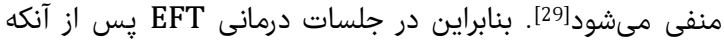

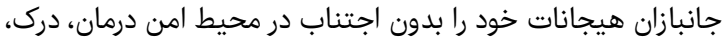

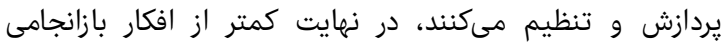

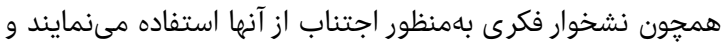

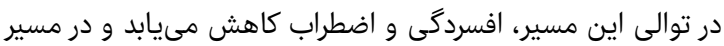

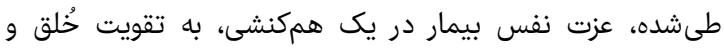

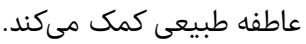

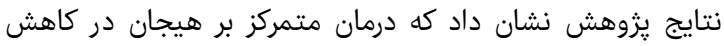

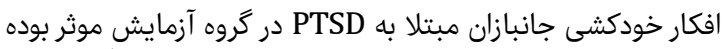

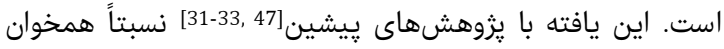

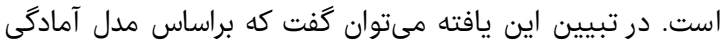

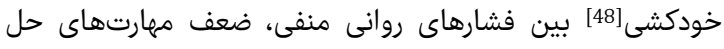

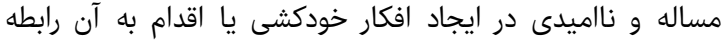

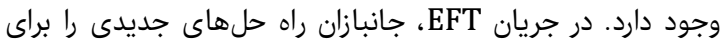

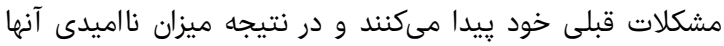
كاهش مىيابد. براساس اين مدل افرادى كئ كه در توانايى تفكر واكرا

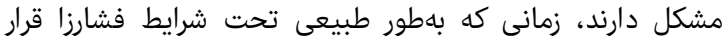

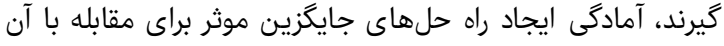

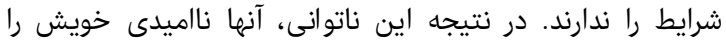

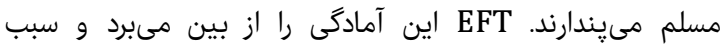

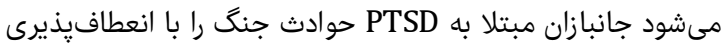

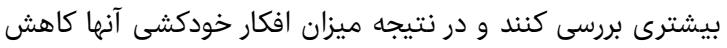

مىيابد.

نتايج يزوهش نشابن داد كه درمان متمركز بر هيجان در نااميدى

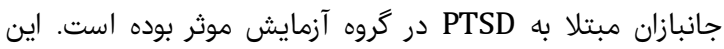

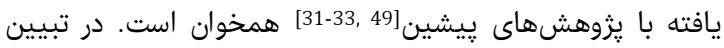

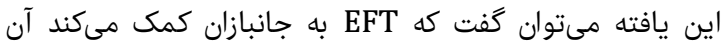

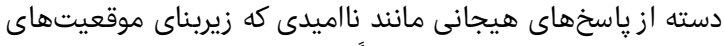

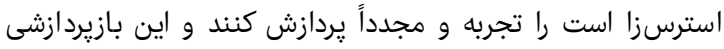

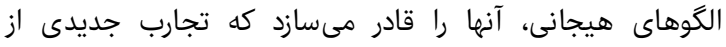

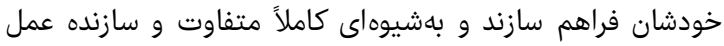

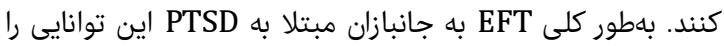

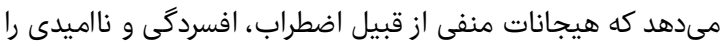

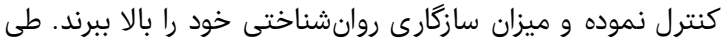

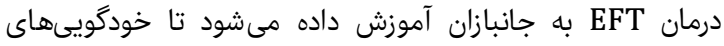

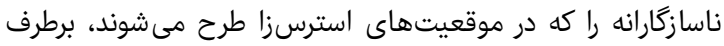

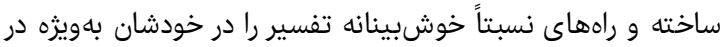

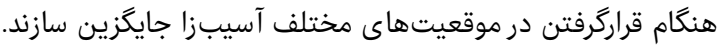

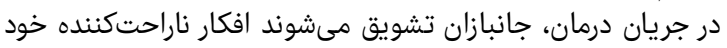

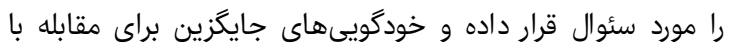

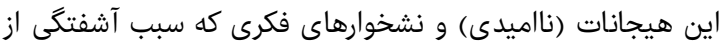

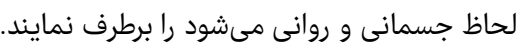

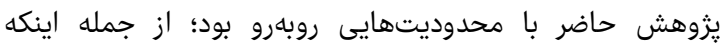

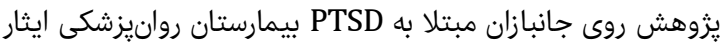

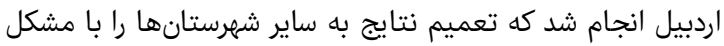

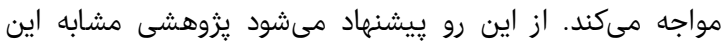

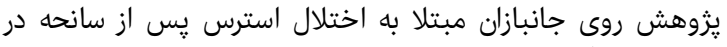

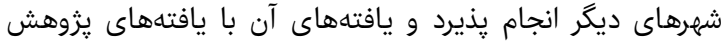

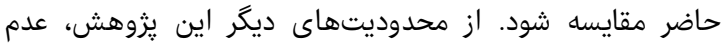

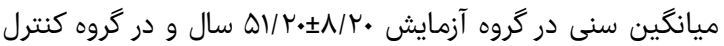

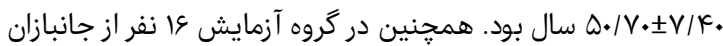

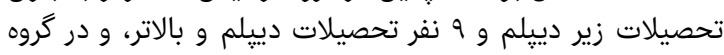

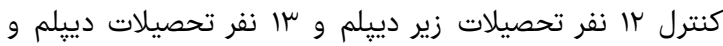
بالاتر داشتند. - مبنر

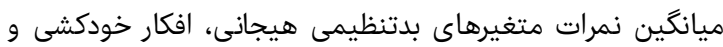

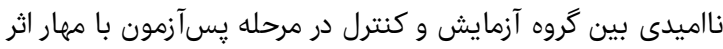

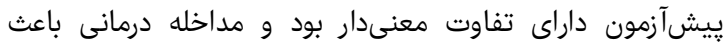

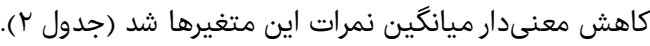

جدول r r مقايسه ميانگين نمرات متغيرهاى بدتنظيمى هيجانى، افكار

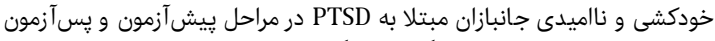

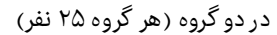

\begin{tabular}{|c|c|c|c|c|}
\hline ضريب اتا & سطح معنىدارى & كروه كنترل & كروه آزمايش & متغيرها \\
\hline & & & \multicolumn{2}{|c|}{ بدتنظيمى هيجانى } \\
\hline \multirow{2}{*}{.1091} & \multirow{2}{*}{.$|\ldots|$} & $\mid F / \Lambda . \pm F / k q$ & $\mid F / 1 . \pm \mu / 9 k$ & ي يشآزمون \\
\hline & & $\mid \Psi / \Delta \Delta \pm \Psi / \Delta$. & $1 \cdot|\Delta \Delta \pm r / \Lambda|$ & يس آزمون \\
\hline & & & \multicolumn{2}{|c|}{ افكار خودكشى } \\
\hline . & $.1 . r$ & $\begin{array}{l}r \Delta / r \varepsilon \pm r / l \varepsilon \\
r \mu / \mu r_{ \pm} r / R\end{array}$ & $\begin{array}{l}r \xi / \mid \Delta \pm r / G . \\
\mid F / \| r \pm r / r F\end{array}$ & يِيش آزمون \\
\hline . IFFA & $.1 . .1$ & $\begin{array}{l}\mid \varepsilon / F \mu_{ \pm} / 1 . \\
|Q / r| \pm r / \mu K\end{array}$ & $\begin{array}{l}\mid N / \Delta \Delta \pm r / q . \\
\| / T \mu \pm I / R \Delta\end{array}$ & يُ \\
\hline
\end{tabular}

بحث

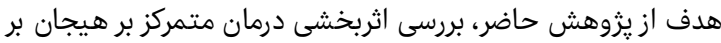

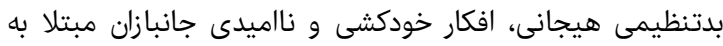

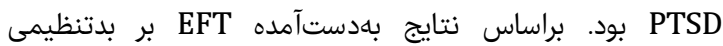

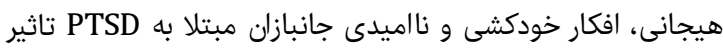

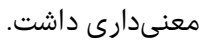
نتايج بزوهش هاري داضر نشان داد درمان متمركز بر هيجان در كاهش دانش

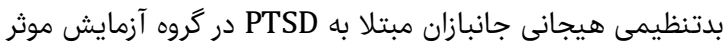

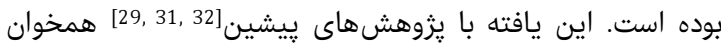

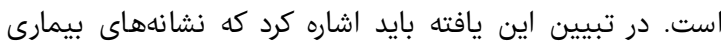

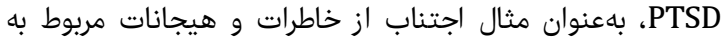

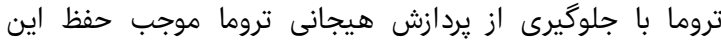

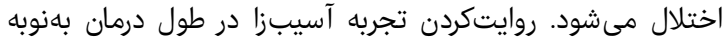

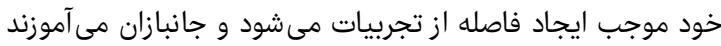

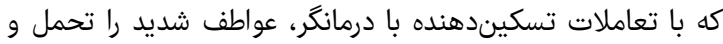

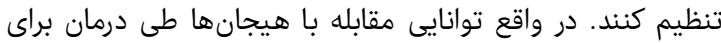

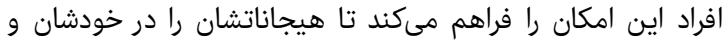

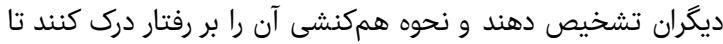

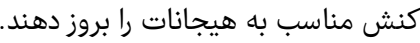

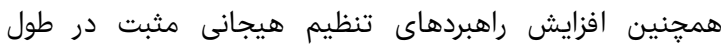

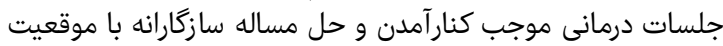

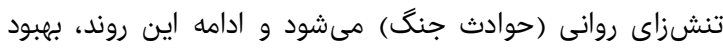

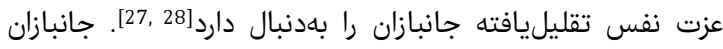

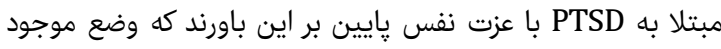

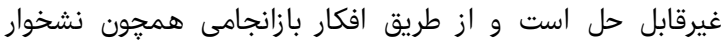

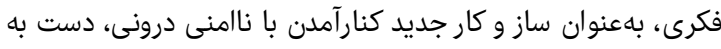

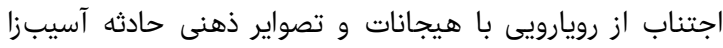

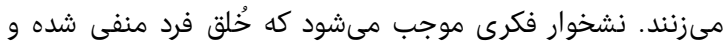
اين روند، آنها را به تجربهكردن هر جه بيش بيشتر نشانكان افسردگى و و 
9- Sheykhan R, Ghazanfari F, Jadidi F, Adineh M, Sadrmohamadi R. Attachment styles and cognitive emotion regulation strategies as predictor of posttraumatic stress disorder in veterans. J Mazand Univ Med Sci. 2016;26(137):95-104. [Persian]

10- krysinska k, Lester D. Post-traumatic stress disorder and suicide risk: A systematic review. Arch Suicide Res. 2010;14(1):1-23.

11- Panagioti M, Gooding PA, Tarrier N. A meta-analysis of the association between posttraumatic stress disorder and suicidality: The role of comorbid depression. Compr Psychiatry. 2012;53(7):915-30.

12- Coohey C, Dirks- Bihun A, M.Renner L, Baller R. Strain, depressed mood and suicidal thoughts among maltreated adolescents in the United States. Child Abuse Negl. 2014;38(7):1171-9.

13- Leiner AS, Compton MT, Houry D, Nadine J, Kaslow J. Intimate partner violence, psychological distress, and suicidality: A path model using data from African American women seeking care in an urban emergency department. J Fam Violence. 2008;23(6):473-81.

14- Pompili M, Sher L, Serafini G, Forte A, Innamorati M, Dominici G, et al. Posttraumatic stress disorder and suicide risk among veterans: A literature review. J Nerv Ment Dis. 2013;201(9):802-12.

15- Ghamari-Givi H, Hosseini Kiasari ST. The effectiveness of cognitive- existential group therapy on hopelessness in patients with multiple sclerosis. Daneshvar. 2014;21(112):1-11. [Persian]

16- Snyder CR. Hope theory: rainbows in the mind. Psychol Inq. 2002;13(4):249-75.

17- Hasson-Ohayon I, Kravetz S, Meir T, Rozencwaig S. Insight into severe mental illness, hope, and quality of life on persons with schizophrenia and schizoaffective disorders. Psychiatry Res. 2009;167(3):231-8.

18- Johnson KL. The relationship of hope and quality of life in combat veterans seeking treatment for posttraumatic stress disorder. Kansas: University of Kansas, Psychology and Research in Education Publisher; 2001. pp. 29-58.

19- Leahy RL. Cognitive therapy techniques: A practitioner s guide. 2rd edition. Fata L, Shakiba Sh, Tahmasbi Moradi Sh, Naseri H, Ziaei K, translators. New York City: Guilford Press; 2001. [Persian]

20- Paunović N. Exposure inhibition therapy as a treatment for chronic posttraumatic stress disorder: A controlled pilot study. Psychology. 2011;2(6):605-14.

21- Talbot NL, Gamble SA. IPT for women with trauma histories in community mental health care. J Contemp Psychother. 2008;38(1):35-44.

22- McCarthy KS, Connolly-Gibbons MB, Barber JP. The relation of rigidity across relationship with symptoms and functioning: An investigation with the revised central relationship questionnaire. J Couns Psychol 2008;55(3):346-58.

23- Fridja NH. The emotions. Cambridge, MA: Cambridge University Press; 1986. pp. 9-41.

24- Greenberg LJ, Warwar SH, Malcolm WM. differential effects of emotion-focused therapy and psychoeducation in facilitating forgiveness and letting go of emotional injuries. J Counsel Psychol. 2008;55(2):185-96.

25- Pos AE, Greenberg LS. Emotion-focused therapy: The trans- forming power of affect. J Contemp Psychother. 2007;37(1):25-31.

26- Greenberg LS, Angus L. The contribution of emotion processes to narrative change: A dialecticalconstructivist approach. In: Angus L, McLeod J, editors.

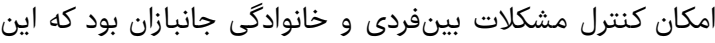

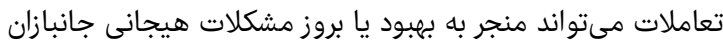

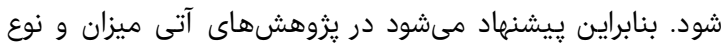

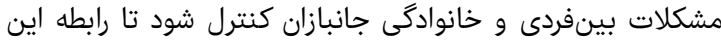

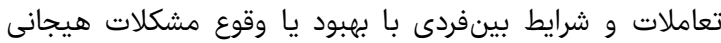

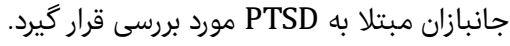

ختيجهه

تشكر و قدردانى: در يايان، نويسندگان اين مقاله از همكارى

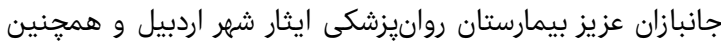

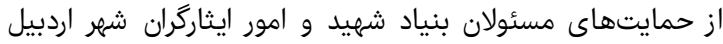

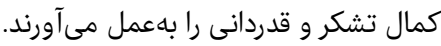

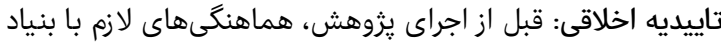

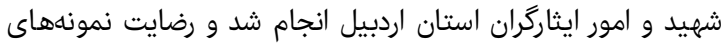

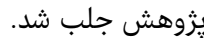
تعارض منافع: موردى از سوى نويسندگان كزارش نشده است.

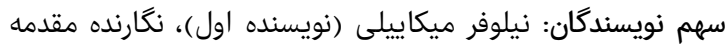

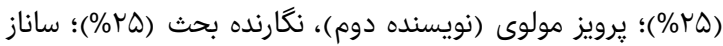

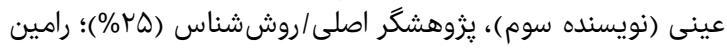

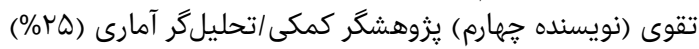

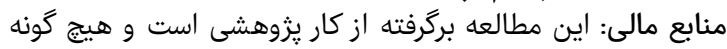

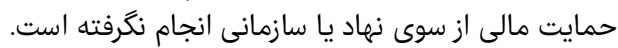

منابع 1- American Psychiatric Association. Diagnostic and statistical manual of mental disorders DSM-5. $5^{\text {th }}$ edition. Rezaei F, Fakhraee A, Charismatic A, Lotus A, Hashemi Azar J, Shamloo F, translators. Virginia, United States: American Psychiatric Association; 2013.

2- Tull MT, Barrett HM, McMillan ES, Roemer L. A preliminary investigation of relationship between emotion regulation difficulties and posttraumatic stress symptoms. Behav Ther. 2007;38(3):303-13.

3- Leahy R, Napolitano LA, Tirch D. Emotion regulation in psychotherapy: A practitioners guide. New York: Guilford Press; 2011.

4- Foa EB, Kozak MJ. Emotional processing of fear: Exposure to corrective information. Psychol Bull. 2008;99(1):20-35.

5- Mazloom M, Yaghubi $H$, Mohammadkhani S. The relationship of metacognitive beliefs and emotion regulation difficulties with posttraumatic stress disorder. J Behav Sci. 2014;8(2):105-13. [Persian]

6- Bardeen JR, Kumpula MJ, Orcutt HK. Emotion regulation difficulties as a prospective predictor of posttraumatic stress symptoms following as mass shooting. J Anxiety Dis. 2013;27(2):188-96.

7- Ozdamir O, Boysan M, Ozdamir PG, Yilmaz E. Relationships between posttraumatic stress disorder (PTSD), dissociation, quality of life, hopelessness, and suicidal ideation among earthquake survivors. Psychiatry Res. 2015;228(2):598-605.

8- Azad Marzabadi E, Moqtadaee K, Aria Pooran S. The effectiveness of mindfulness training on psychological symptoms in veterans with post- traumatic stress disorder. J Behav Sci. 2013;7(1):67-74. [Persian] 
IIV اثربخشى درمان متمركز بر هيجان بر بدتنظيمى هيجانى، افكار خودكشى و ناميدى در جانبازان مبتلا به اختلال استرس يس سانحه

of research. Depress Anxiety. 2001;13(3):132-56.

38- Mirzaee J, Karami GR, Ameli J, Hemmati MA. Investigation of clinical diagnosis by psychological tests in PTSD outpatients and inpatients. J Mil Med. 2004;6(3):201-8. [Persian]

39- Goodarzi MA. Reliability and validity of post- traum atic stress disorder Mississippi scale. J Psychol. 2003;7(2):135-78. [Persian]

40-Beck AT, Weissman A, Lester D. The measurement of pessimism: the hopelessness scale. J Consult Clin Psychol. 1974;42(6):861-5.

41- Dejhkam N, Sharifi HP, Human HA. Conformity and norm of the beck hopelessness scale among students of Tehran Islamic Azad University [Dissertation]. Tehran: Tehran University; 2003. [Persian]

42- Yousefi N, Shirbigy N. The relationship between acceptation in ma exam with hopelessness and selfhandicapping. Iran High Educ. 2012;4(4):159-81. [Persian]

43- Kaviani H. Interview and psychological inventories. $1^{\text {st }}$ edition. Tehran: Besat Press; 2002. pp. 171-8. [Persian]

44- Gratz KL, Roemer L. Multidimensional assessment of emotion regulation a dysregulation: development, factor structure, and initial validation of the difficulties in emotion regulation scale. J Psychopathol Behav Assess. 2008;30(4):315-9.

45- Asgari P, Pasha G, Aminian M. The relationship emotional regulation, life events and body image with eating disorder in women. Andisheh va Raftar. 2009;4(13):65-78. [Persian]

46- Greenberg LS. Emotion-Focused Therapy: A Clinical Synthesis. J Lifelong Learn Psychiatry. 2010;8(1):32-42.

47- Sabury M. The effectiveness of cognitive therapy in reducing suicidal thoughts and emotion-focused cognitive triangle patients with major depression [Dissertation]. Mashhad: Mashhad University; 2013. [Persian]

48- Schotte DE, Clum GA. Problem- solving skills in suicidal psychiatric patients. J Consult Clin Psychol. 1987;55(1):49-54.

49- Narimani M, Alisari Nasirlou K, Effat Parvar S. The effectiveness of emotion-focused training for cognitive emotional regulation strategies of emotionally abused students. Appl Couns. 2013;3(2):37-50. [Persian]
Handbook of narrative and psychotherapy: Practice, theory, and research. Thousand Oaks, CA, US: Sage Publications; 2004. pp. 331-50.

27- Greenberg LS. Emotion focused therapy for depression. Pers Exp Psychother. 2017;16(2):106-17.

28- Prochaska JO, Norcross JC. Systems of psychotherapy. Seyyed Mohammadi Y, translator. Pacific Grove: Brooks Cole; 2009.

29- Greenberg LS, Foerster F. Task analysis exemplified: The process of resolving unfinished business. J Cons Clin Psychol. 1996;64(3):439-46.

30- Greenberg LS, Paivio SC. Working with the emotions in psychotherapy. New York City: Guilford Press; 1997. pp. 14-290.

31- Church D. The treatment of combat trauma in veterans using EFT (Emotional Freedom Techniques): A pil ot protocol. J Traumatology. 2010:16(1):55-65.

32- Church D, Hawk C, Brooks AJ, Toukolehto O, Wren M, Dinter I, et al. Psychological trauma symptom improvement in veterans using emotional freedom techniques: A randomized controlled trial. J Nerv Ment Dis.. 2013;201(2):153-60.

33- Akbari E, Poursharifi H, Fahimi S, Azimi Z, Alilou MM, Amiri Pichakolaei A, et al. Effectiveness of emotion focused therapy on sexual victims of romantic relationships: A single case study. J Fundam Ment Health. 2016;18(1):10-21. [Persian]

34- Khojasteh Mehr R, Shiralinia KH, Rajabi GH, Beshlideh K. The effects of emotion-focused couple therapy on depression symptoms reduction and enhancing emotional regulation in distressed couples. Appl Couns. 2012;3(1):1-18. [Persian]

35- Sobhi-Gharamaleki N, Porzoor P, Aghajani S, Narimani M. Effectiveness of emotion regulation training on reduction of anxiety, stress and depression symptoms among university students. Health Educ Health Promot. 2015;3(1):5-13. [Persian]

36- Golpour R, Abolghasemi A, Ahadi B, Narimani M. The effectiveness of cognitive self-compassion training and emotion-focused therapy on quality of life with depression disorder. J Clin Psychol. 2014;1(21):53-64. [Persian]

37- Weathers FW, Keane TM, Davidson JR. Clinicianadministered PTSD scale: A review of the first ten years 